\title{
Re: Association between preconception paternal smoking and birth defects in offspring: evidence from the database of the National Free Preconception Health Examination Project in China. (First comment on BJOG-19-1709.R1)
}

\author{
Jania $\mathrm{Wu}^{1}$, Kyla Ng Yin², Keng Siang Lee ${ }^{3}$, and John Zhang ${ }^{1}$ \\ ${ }^{1}$ National University Singapore Yong Loo Lin School of Medicine \\ ${ }^{2}$ Barts and The London School of Medicine and Dentistry \\ ${ }^{3}$ Bristol Medical School
}

June 23, 2020

Sir ,

We read with great interest the study by Zhou et al. ${ }^{1}$, where the authors demonstrated a significant association between preconception paternal smoking and birth defects in the offspring. The study is timely given the increasing recognition of the role that paternal factors play in pregnancy outcomes. The authors must be congratulated on performing a large scale, nationwide study, in which a potential link between preconception paternal smoking and birth defects in offspring was made.

A salient point discussed by Zhou and colleagues is the difficulty in estimating the effect of paternal smoking as an independent variable on the risk of birth defects due to a myriad of confounding factors. Although the authors had adequately adjusted for maternal biological, environmental and behavioural factors, as well as paternal alcohol consumption, it may be prudent to note that paternal age could be a potentially significant confounder in this relationship.

Advanced paternal age has shown robust links with increases in sperm DNA fragmentation. ${ }^{2}$ DNA damage is attributed to environmental, hormonal and degenerative changes. ${ }^{2}$ Over time, multiple cycles of mitotic replications generate greater stress on the DNA repair mechanisms. ${ }^{2}$ The failure to control the DNA repair mechanisms invariably amounts to ejaculated spermatozoa containing a higher proportion of abnormal paternal DNA. ${ }^{2}$ In the same vein, children born to fathers of advanced age are at a slightly increased risk of birth defects including cardiac, respiratory, gastrointestinal tract and musculoskeletal abnormalities. ${ }^{3}$ It has been speculated that mutations accumulate over repeated spermatogenesis, subsequently increasing the number of birth defects in the offspring. ${ }^{3}$ At the opposite end of the age spectrum, young paternal age has also been associated with a slight increased risk of selected birth defects. ${ }^{3}$

At large, prenatal smoke exposure has unfavourable effects on pregnancies. Effects of maternal smoking has been comprehensively studied and is associated with fetal and developmental conditions. Harmful substances in tobacco smoke such as nicotine, carbon monoxide and polycyclic aromatic hydrocarbons are able to cross the placenta and negatively affect the fetal development. ${ }^{4}$ On the other hand, the extent of paternal smoking has not been well-established. Exposure to cigarette smoke has been found to affect sperm parameters, as well as increase sperm chromatin structural abnormalities and DNA damage. ${ }^{5}$ Infant low birth weight, increased obesity risk in childhood and high blood pressure have been identified as potential complications of paternal smoking. ${ }^{4}$ This study provides new evidence that birth defects are also complications of paternal smoking. 
In conclusion, Zhou et al. have accomplished a tremendous job of investigating the effects of paternal smoking on birth defects. The current study has laid the groundwork for future research to be built upon and to elucidate the true effects of paternal smoking during pregnancy. As exposure to first or second-hand smoke poses a major risk to pregnancies, it might be worthwhile to recommend smoking cessation in both parents during preconception counselling.

Jania J. Y. Wu ${ }^{1}$, Kyla Ng Yin², Keng Siang Lee ${ }^{3}$, John J. Y. Zhang ${ }^{1}$

${ }^{1}$ Yong Loo Lin School of Medicine, National University of Singapore, Singapore

${ }^{2}$ Barts and the London School of Medicine and Dentistry, Queen Mary University of London, London, UK

${ }^{3}$ Bristol Medical School, Faculty of Health Sciences, University of Bristol, Bristol, UK

References

1. Zhou Q, Zhang S, Wang Q, Shen H, Zhang Y, Tian W, et al. Association between preconception paternal smoking and birth defects in offspring: evidence from the database of the National Free Preconception Health Examination Project in China. Bjog. 2020 Apr 27.

2. Humm KC, Sakkas D. Role of increased male age in IVF and egg donation: is sperm DNA fragmentation responsible? Fertil Steril. 2013 Jan;99(1):30-6.

3. Yang Q, Wen SW, Leader A, Chen XK, Lipson J, Walker M. Paternal age and birth defects: how strong is the association? Hum Reprod. 2007 Mar;22(3):696-701.

4. Banderali G, Martelli A, Landi M, Moretti F, Betti F, Radaelli G, et al. Short and long term health effects of parental tobacco smoking during pregnancy and lactation: a descriptive review. J Transl Med. 2015 Oct 15;13:327.

5. Jenkins TG, James ER, Alonso DF, Hoidal JR, Murphy PJ, Hotaling JM, et al. Cigarette smoking significantly alters sperm DNA methylation patterns. Andrology. 2017 Nov;5(6):1089-99. 